JOURNAL OF THE

AMERICAN MATHEMATICAL SOCIETY

Volume 11, Number 3, July 1998, Pages 635-641

S 0894-0347(98)00263-X

\title{
L-SERIES WITH NONZERO CENTRAL CRITICAL VALUE
}

\author{
KEVIN JAMES
}

\section{INTRODUCTION}

Suppose that $f=\sum_{n \geq 1} a_{n}(f) q^{n}$ is a cusp form of weight $2 k(k \in \mathbb{N})$. We denote by $L(f, s)$ the $L$-function of $f$. For $\operatorname{Re}(s)$ sufficiently large, the value of $L(f, s)$ is given by $L(f, s)=\sum_{n \geq 1} \frac{a_{n}(f)}{n^{s}}$, and one can show that $L(f, s)$ has analytic continuation to the entire complex plane. The value of $L(f, s)$ at $s=k$ will be of particular interest to us, and we will refer to this value as the central critical value of $L(f, s)$.

Let $\chi_{D}$ denote the Dirichlet character associated to the extension $\mathbb{Q}(\sqrt{D}) / \mathbb{Q}$, that is, $\chi_{D}(n)=\left(\frac{\Delta_{D}}{n}\right)$, where $\Delta_{D}$ denotes the discriminant of $\mathbb{Q}(\sqrt{D}) / \mathbb{Q}$. Define the $D^{\text {th }}$ quadratic twist of $f$ to be $f_{\chi_{D}}=\sum_{n \geq 1} a_{n}(f) \chi_{D}(n) q^{n}$. For any integer $D$, the $L$-function of $f_{\chi_{D}}$ is the $D^{\text {th }}$ quadratic twist of $L(f, s)$, that is, $L\left(f_{\chi_{D}}, s\right)=$ $\sum_{n \geq 1} \frac{a_{n}(f) \chi_{D}(n)}{n^{s}}$. We will be interested in determining how often $L\left(f_{\chi_{D}}, s\right)$ has nonzero central critical value as $D$ varies over all integers. Since $\chi_{D m^{2}}=\chi_{D}$, we will restrict our attention to the square-free integers $D$. We expect that as we let $D$ vary over all of the square-free integers, a positive proportion of the $L$-functions $L\left(f_{\chi_{D}}, s\right)$ will have nonzero central critical value. Indeed, Goldfeld [7] conjectures that for newforms $f$ of weight $2, L\left(f_{\chi_{D}}, 1\right) \neq 0$ for $\frac{1}{2}$ of the square-free integers.

Given an elliptic curve $E: y^{2}=x^{3}+A x^{2}+B x+C(A, B, C \in \mathbb{Z})$ with conductor $N_{E}$ and an integer $D$, we define the $D^{\text {th }}$ quadratic twist of $E$ to be the curve $E_{D}: y^{2}=x^{3}+A D x^{2}+B D^{2} x+C D^{3}$. Let $L\left(E_{D}, s\right)$ denote the $L$-function associated to $E_{D}$. For square-free $D$ coprime to $2 N_{E}, L\left(E_{D}, s\right)$ is simply the $D^{\text {th }}$ quadratic twist of $L\left(E_{1}, s\right)$.

If $f \in S_{2}(N)$ is a newform with integer coefficients, we know via the theory of Eichler and Shimura that there is an elliptic curve $E$ over $\mathbb{Q}$ having conductor $N$ so that $L(E, s)=L(f, s)$. Thus if $D$ is coprime to $2 N$, then $L\left(E_{D}, s\right)=L\left(f_{\chi_{D}}, s\right)$. Also, one knows from the work of Kolyvagin [13], as supplemented by the work of Murty and Murty [17] or that of Bump, Friedberg and Hoffstein [3] (see also [10] for a shorter proof), that if $E$ is a modular elliptic curve and if $L(E, 1) \neq 0$, then the rank of $E$ is 0 . Thus, if $f$ has the property that a positive proportion of the twists of $L(f, s)$ have nonzero central critical value, then this implies that a positive density of the quadratic twists $E_{D}$ have rank 0 .

There have been many papers which have proved results in this direction. For example, in [2], [3], [6], [10], [16], [17], [19], [28] one can find general theorems on

Received by the editors August 13, 1997 and, in revised form, January 20, 1998.

1991 Mathematics Subject Classification. Primary 11G40.

(C)1998 American Mathematical Society 
the vanishing and nonvanishing of the quadratic twists of a given $L$-function. These theorems ensure that an infinite number of the quadratic twists of an $L$-function associated to a cusp form will have nonzero central critical value.

In [20], Ono has shown several examples of cusp forms $f$ associated to elliptic curves such that for a positive density of the primes $p$, the $p^{\text {th }}$ quadratic twist of $L(f, s)$ will have nonzero central critical value. Ono also proves a theorem which gives sufficient conditions under which a cusp form associated to an elliptic curve will have this property. Using methods similar to those of Ono, the author [11] was able to prove that the elliptic curve $E_{p}: y^{2}=x^{3}-32 p^{3}$ has rank 0 for at least $1 / 3$ of the primes $p$.

Subsequently, Ono and Skinner [22] used the theory of Galois representations to extend Ono's theorem to all even weight eigenforms satisfying a very mild hypothesis. In fact they verify that this hypothesis is satisfied for all modular elliptic curves of conductor less than or equal to 100 .

In a series of two papers [8], [9], Heath-Brown has done an extensive investigation of the behavior of the 2-Selmer groups associated to the quadratic twists of the congruent number curve: $y^{2}=x^{3}-x$. He states as a corollary to one of his theorems that at least $5 / 16$ of these quadratic twists have rank 0 . This result along with the Birch and Swinnerton-Dyer conjecture implies that at least 5/16 of the quadratic twists of the $L$-function $L(E, s)$ associated to the congruent number curve should have nonzero central critical value.

Using ideas developed by Frey [5] along with the Davenport-Heilbronn theorem [18], Wong [27] has shown the existence of an infinite family of nonisomorphic elliptic curves such that a positive proportion of the quadratic twists of each curve have rank 0. Thus if we assume the Birch and Swinnerton-Dyer and ShimuraTaniyama conjectures, Wong's result would then imply the existence of an infinite family of weight 2 cusp forms $\left\{f_{i}\right\}$ such that a positive proportion of the twists of each $L\left(f_{i}, s\right)$ have nonzero central critical value.

In section 3 of this paper we exhibit weight 2 newforms $F$ such that $L\left(F_{\chi_{D}}, 1\right) \neq 0$ for all $D$ in a subset of the square-free natural numbers having positive lower density. We now describe the first of those results. Let $E$ denote the elliptic curve with equation $y^{2}=x^{3}-x^{2}+72 x+368$. Then $E$ is a modular curve (it is the -1 twist of $X_{0}(14)$ ). We let $F$ denote the weight 2 cusp form whose Mellin transform is $L(E, s)$. We then prove unconditionally:

Theorem 1. $L\left(F_{\chi_{D}}, 1\right) \neq 0$ for at least $7 / 64$ of the square-free natural numbers $D$.

In light of Kolyvagin's work, we have as a corollary to Theorem 1

Corollary 2. For at least $7 / 64$ of the square-free natural numbers $D, E_{D}$ has rank 0 .

Our proof differs from those of Heath-Brown and Wong in that while they work directly with the Selmer groups of elliptic curves, our proof uses the theory of modular forms developed by Shimura and Waldspurger to gain information about the central critical values of the $L$-functions associated to elliptic curves. An outline of the proof of Theorem 1 is as follows. Using ideas of Schoeneberg [23] and Siegel [25], we construct a weight $3 / 2$ cusp form $f$ as the difference of the theta functions associated to two inequivalent ternary quadratic forms $Q_{1}$ and $Q_{2}$ which together make up a genus of ternary forms. This $f$ will be an eigenform for all of the 
Hecke operators and will lift through the Shimura correspondence to $F_{\chi_{-1}}$. By a theorem of Waldspurger [26] we will be able to equate the vanishing of the central critical values of the quadratic twists of $L(F, s)$ to the vanishing of certain Fourier coefficients of $f$. Since our ternary forms $Q_{1}$ and $Q_{2}$ are the only forms in a certain genus of ternary forms, we are able to study the automorph structure of these forms to show that the Fourier coefficients of $f$ are related modulo 3 to certain class numbers of imaginary quadratic number fields. We will then use the DavenportHeilbronn theorem (see [18]) to show that at least $7 / 64$ of these class numbers are not divisible by 3 , and hence, the associated Fourier coefficients of $f$ are nonzero. It will then follow that at least $7 / 64$ of the quadratic twists of $L(F, s)$ have nonzero central critical value.

The key ingredient in the above argument is that our ternary forms $Q_{1}$ and $Q_{2}$ are a complete set of representatives for a genus of forms having the correct automorph structure. In particular, if $A_{i}$ denotes the number of automorphs of $Q_{i}(i=1,2)$, then we need that $A_{1}+A_{2} \equiv 0$ modulo 3 while $3 \nmid A_{1} A_{2}$. This congruence modulo 3 is what allows the use of the Davenport-Heilbronn Theorem. It is important to note that there are examples of genera of ternary quadratic forms which contain exactly two equivalence classes but whose automorph structure does not satisfy the above congruence modulo 3 (see the tables of ternary forms in [14]) and, for such examples, the Davenport-Heilbronn Theorem is of no consequence.

\section{BACKGROUND}

The theory developed by Waldspurger in [26] provides a tool for obtaining information about the central critical values of the $L$-series $L\left(f_{\chi_{n}}, s\right)$ associated to the quadratic twists of a particular integral weight newform $f$. Before stating his results we need to introduce one more bit of notation. If $f$ is a newform of weight $2 k$ and if $\chi$ is a Dirichlet character, then $f_{\chi}$ is an eigenform for all of the Hecke operators. Hence, by the theory of newforms developed in [1] and [15], there exists a newform of weight $2 k$ which, following Waldspurger, we will denote $f \cdot \chi$ with the same eigenvalues as $f_{\chi}$ for all but finitely many of the Hecke operators. In fact it is the central critical values of the $L\left(f \cdot \chi_{n}, s\right)$ which Waldspurger's theorem allows us to relate to the Fourier coefficients of a half-integral weight form. Since $f_{\chi_{n}}$ and $f \cdot \chi_{n}$ have the same eigenvalues for all but a finite number of the Hecke operators, it follows that $L\left(f \cdot \chi_{n}, s\right)$ and $L\left(f_{\chi_{n}}, s\right)$ differ only by a finite number of Euler factors. Thus, $L\left(f_{\chi_{n}}, k\right)=0$ if and only if $L\left(f \cdot \chi_{n}, k\right)=0$.

Now, we are ready to state a special case of the main theorem in [26].

Theorem 2.1. Let $k \geq 3$ be an odd integer, $N \in 4 \mathbb{N}, \chi$ a Dirichlet character modulo $N$, and $M$ some divisor of $N$ so that $\chi^{2}$ is a Dirichlet character modulo $M$. Suppose $F \in S_{k-1}\left(M, \chi^{2}\right)$ is a newform with Hecke eigenvalues $\lambda_{p}(F)$. Suppose also that there exists a cusp form $f \in S_{k / 2}(N, \chi)$ having the property that for all but finitely many primes $p, T_{p} f=\lambda_{p}(F) f$. Finally suppose that the Dirichlet character $\nu$ defined by $\nu(n)=\chi(n)\left(\frac{-1}{n}\right)^{\frac{k-1}{2}}$ has conductor divisible by 4 . Let $\mathbb{N}^{\text {sf }}$ denote the square-free natural numbers. Then there is a function $\mathbb{A}: \mathbb{N}^{s f} \rightarrow \mathbb{C}$, depending only on $F$ and satisfying the condition

$$
(\mathbb{A}(t))^{2}=L\left(F \cdot \nu^{-1} \chi_{t}, \frac{k-1}{2}\right) \cdot \epsilon\left(\nu^{-1} \chi_{t}, 1 / 2\right),
$$


where $\epsilon(\psi, s)$ is chosen so that if $L(\psi, s)$ is the Dirichlet $L$-function for the Dirichlet character $\psi$ and if

$$
\Lambda(\psi, s)= \begin{cases}\pi^{-s / 2} \Gamma\left(\frac{s}{2}\right) L(\psi, s) & \text { if } \psi(-1)=1, \\ \pi^{-(s+1) / 2} \Gamma\left(\frac{s+1}{2}\right) L(\psi, s) & \text { if } \psi(-1)=-1,\end{cases}
$$

then

$$
\Lambda\left(\psi^{-1}, 1-s\right)=\epsilon(\psi, s) \Lambda(\psi, s)
$$

Moreover $f$ can be written as a finite $\mathbb{C}$-linear combination of Hecke eigenforms $f_{i}$ such that $a_{t}\left(f_{i}\right)=c\left(t^{\mathrm{sf}}, F\right) \mathbb{A}(t)$, where $t^{\mathrm{sf}}$ denotes the square-free part of $t$ and $c\left(t^{\mathrm{sf}}, F\right) \in \mathbb{C}$.

In particular, we can deduce from Theorem 2.1 that if $a_{t}(f) \neq 0$, then

$$
L\left(F \cdot \nu^{-1} \chi_{t}, \frac{k-1}{2}\right) \neq 0 .
$$

Also, we will use the following theorem which is an immediate corollary of a theorem of Davenport and Heilbronn [4] as improved by Nakagawa and Horie [18].

Theorem 2.2. Suppose that $m$ and $N$ satisfy:

1. If $p$ is an odd prime dividing $(N, m)$, then $p^{2} \mid N$ and $p^{2} \nmid m$, and

2. If $N$ is even, then either $4 \mid N$ and $m \equiv 1$ modulo 4 or $16 \mid N$ and $m \equiv 8$ or 12 modulo 16.

Let $T$ denote the set of discriminants $\Delta$ of imaginary quadratic extensions of $\mathbb{Q}$ in the arithmetic progression $\Delta \equiv m$ modulo $N$. Then there is a subset $S$ of $T$ having lower density at least $\frac{1}{2}$ in $T$ such that if $\Delta \in S$, then $3 \nmid h(\Delta)$.

\section{NONVANISHING THEOREMS}

If $Q$ is a primitive positive definite ternary quadratic form, then we will denote the discriminant of $Q$ by $d_{Q}$. We also define $\theta_{Q}(\tau)=\sum_{x, y, z \in \mathbb{Z}} q^{Q(x, y, z)}\left(q=e^{2 \pi i \tau}\right)$. It is well known (see [24]) that $\theta_{Q}$ is a modular form of weight $3 / 2$, and we have a theorem of Siegel [25] which states that if $Q_{1}$ and $Q_{2}$ are two primitive positive definite ternary quadratic forms belonging to the same genus, then $\left(\theta_{Q_{1}}-\theta_{Q_{2}}\right)$ is a cusp form.

Proposition 3.1. Suppose that $Q_{1}$ and $Q_{2}$ are even integral primitive positive definite ternary quadratic forms and that $Q_{1}$ and $Q_{2}$ are the only forms in a genus of forms. Let $A_{i}$ denote the number of automorphs of $Q_{i}(i=1,2)$. Assume that $3 \nmid A_{1} A_{2}$ but $3 \mid A_{1}+A_{2}$. Suppose also that $f=\left(\theta_{Q_{1}}-\theta_{Q_{2}}\right) \in S_{3 / 2}\left(N, \chi_{q}\right)$ is a Hecke-eigenform which lifts through the Shimura correspondence to a cusp form $F \in S_{2}(N / 2)$. Let $G$ denote the unique weight 2 newform of trivial character having $\lambda_{p}(F)=\lambda_{p}(G)$ for all but finitely many of the primes $p$, and let $N_{G}$ denote the level of $G$. Put

(1)

$R=\left\{a \in\left(\mathbb{Z} / 4 d_{Q_{1}}^{\mathrm{sf}} \mathbb{Z}\right)^{*}: \exists\right.$ a square-free $n \equiv a \quad(\bmod 8 W)$ with $\left.3 \nmid a_{n}(f)\right\} \quad$ and, $\delta=\frac{\# R}{8 d_{Q_{1}}^{\text {sf }} \prod_{p \mid d_{Q_{1}}^{\text {sf }}}\left(1-\frac{1}{p^{2}}\right)}$.

Then, the set of square-free natural numbers $n$ such that $L\left(G \cdot \chi_{-q n}, 1\right) \neq 0$ has lower density at least $\delta$ in the square-free natural numbers. 
Proof. Let $R\left(Q_{1}, m\right)$ denote the number of essentially distinct primitive representations of $m$ by the genus of ternary forms containing $Q_{1}$ and let $r_{i}(m)$ denote the total number of representations of $m$ by $Q_{i}(i=1,2)$. Then for sufficiently large square-free natural numbers $m$, we have $R\left(Q_{1}, m\right)=\frac{r_{1}(m)}{A_{1}}+\frac{r_{2}(m)}{A_{2}}$.

Now, suppose that $a \in R$. Then there exists $n \equiv a$ modulo $4 d_{Q_{1}}^{\text {sf }}$ such that $3 \nmid a_{n}(f)$; hence, it follows from the construction of $f$ that $R\left(Q_{1}, n\right) \neq 0$. Thus, $R\left(Q_{1}, m\right) \neq 0$ for all natural numbers $m \equiv a$ modulo $4 d_{Q_{1}}^{\text {sf }}$. Applying a theorem of Gauss (see [12, Theorem 86]) and recalling the relationship of the class number of an order in an imaginary quadratic field to the class number of the ring of integers in the same field, we have that for all square-free natural numbers $m \equiv a$ modulo $4 d_{Q_{1}}^{\mathrm{sf}}$,

$$
R\left(Q_{1}, m\right)=\rho h\left(\Delta_{-m}\right),
$$

where $\rho \in \mathbb{Q}$. Since $3 \nmid A_{1} A_{2}$ and $3 \mid A_{1}+A_{2}$, we have that $A_{1} A_{2} R\left(Q_{1}, m\right)=$ $A_{2} r_{1}(m)+A_{1} r_{2}(m) \equiv A_{2}\left(r_{1}(m)-r_{2}(m)\right)$ modulo 3 . From our construction of $f$, we have that $a_{m}(f)=r_{1}(m)-r_{2}(m)$. Therefore, $3 \mid a_{m}(f)$ if and only if $3 \mid \rho h\left(\Delta_{-m}\right)$. Recall that $3 \nmid a_{n}(f)$ and $n \equiv a$ modulo $4 d_{Q_{1}}^{\text {sf }}$, and therefore $3 \nmid \rho h\left(\Delta_{-n}\right)$. Thus, we see that $\operatorname{ord}_{3}(\rho) \leq 0$. By the Davenport-Heilbronn Theorem (Theorem 2.2), we have for at least half of the square-free natural numbers $m \equiv a$ modulo $4 d_{Q_{1}}^{\text {sf }}$ that $3 \nmid h\left(\Delta_{-m}\right)$. Therefore, we have that $\operatorname{ord}_{3}(\rho)=0$ and hence it follows for all square-free natural numbers $m \equiv a$ modulo $4 d_{Q_{1}}^{\text {sf }}$ that $3 \mid a_{m}(f)$ if and only if $3 \mid h\left(\Delta_{-m}\right)$. Now, applying Theorem 2.2 again, we see for each $a \in R$ that $a_{m}(G) \neq 0$ for at least $1 / 2$ of the square-free natural numbers $m \equiv a$ modulo $4 d_{Q_{1}}^{\text {sf }}$, and hence by Theorem 2.1 that $L\left(G \cdot \chi_{-q m}, 1\right) \neq 0$. We note that each $a \in R$ gives rise to $d_{Q_{1}}^{\text {sf }}$ arithmetic progressions modulo $4\left(d_{Q_{1}}^{\text {sf }}\right)^{2}$, and that the total number of arithmetic progressions modulo $4\left(d_{Q_{1}}^{\text {sf }}\right)^{2}$ in which square-free numbers reside is $4\left(d_{Q_{1}}^{\text {sf }}\right)^{2} \prod_{p \mid d_{Q_{1}}^{\text {sf }}}\left(1-\frac{1}{p^{2}}\right)$. Thus the density of square-free natural numbers $m$ which are congruent modulo $4 d_{Q_{1}}^{\mathrm{sf}}$ to some $a \in R$ is $\frac{\# R \cdot d_{Q_{1}}^{\text {sf }}}{4\left(d_{Q_{1}}^{\text {sf }}\right)^{2} \prod_{p \mid d_{Q_{1}}}^{\text {sf }}\left(1-\frac{1}{p^{2}}\right)}$. The proposition now follows from Theorem 2.2.

Example 3.1. Let

$$
\begin{aligned}
& Q_{1}(x, y, z)=x^{2}+7 y^{2}+7 z^{2}, \text { and } \\
& Q_{2}(x, y, z)=2 x^{2}+4 y^{2}+7 z^{2}-2 x y .
\end{aligned}
$$

Then one can easily check that $Q_{1}$ and $Q_{2}$ both have discriminant 196 and that they are both in the same genus. The numbers of automorphs of $Q_{1}$ and $Q_{2}$ are 8 and 4 respectively. Also, we can calculate (see [14] or [24]) that $\theta_{Q_{1}}, \theta_{Q_{2}} \in M_{3 / 2}(28)$, and therefore by [25] we have that $f=\left(\theta_{Q_{1}}-\theta_{Q_{2}}\right) \in S_{3 / 2}(28)$. We checked computationally that $f$ is an eigenform for all of the Hecke operators and that $f$ lifts through the Shimura correspondence to twice the weight 2 newform $F$ of level 14 associated to the elliptic curve $E: y^{2}=x^{3}+x^{2}+72 x-368$ of conductor 14, that is, $L(F, s)=L(E, s)$. Thus, $f$ satisfies the hypotheses of Proposition 3.1. In this case, we have $d_{Q_{1}}^{\text {sf }}=14$, and by computing the first 200 coefficients of $f$, we see that $1,9,15,23,25,29,37,39$ and 53 are all elements of $R$. Therefore, $\delta=7 / 64$, and Theorem 1 now follows from Proposition 3.1.

We were also able to obtain positive density nonvanishing results as in Corollary 2 for the quadratic twists of nine other elliptic curves. Since the calculations involved 
in the verification of the hypotheses of Proposition 3.1 are completely analogous to the calculations discussed in Example 3.1, we omit them and simply present the results in the following table. We list for each curve $E$ a Weierstrauss equation for $E$, the conductor $N_{E}$ of $E$, and the lower bound $\delta_{E}$ on the lower density of square-free natural numbers $d$ such that $L\left(E_{-d}, 1\right) \neq 0$.

\begin{tabular}{|c|c|c|}
\hline$E$ & $N_{E}$ & $\delta_{E}$ \\
\hline$y^{2}=x^{3}+x^{2}+72 x-368$ & 14 & $7 / 64$ \\
$y^{2}=x^{3}+8$ & 576 & $1 / 4$ \\
$y^{2}=x^{3}+1$ & 36 & $5 / 24$ \\
$y^{2}=x^{3}+4 x^{2}-144 x-944$ & 19 & $19 / 240$ \\
$y^{2}=x^{3}+x^{2}+4 x+4$ & 20 & $5 / 72$ \\
$y^{2}=x^{3}+x^{2}-72 x-496$ & 26 & $13 / 112$ \\
$y^{2}=x^{3}+x^{2}+24 x+144$ & 30 & $5 / 128$ \\
$y^{2}=x^{3}+x^{2}-48 x+64$ & 34 & $17 / 144$ \\
$y^{2}=x^{3}+x^{2}+3 x-1$ & 44 & $11 / 144$ \\
$y^{2}=x^{3}+5 x^{2}-200 x-14000$ & 50 & $5 / 24$ \\
\hline
\end{tabular}

\section{ACKnowledgments}

The author wishes to thank Ken Ono for many insightful discussions and for the suggestion of attacking this problem by studying the automorph structure of ternary forms. He would also like to thank Andrew Granville and David Penniston for their help during the preparation of this manuscript.

\section{REFERENCES}

1. A.O.L. Atkin and J. Lehner, Hecke operators on $\Gamma_{0}(m)$, Math. Ann. 185 (1970), 134-160. MR 42:3022

2. D. Bump, S. Friedberg, and J. Hoffstein, Eisenstein series on the metaplectic group and nonvanishing theorems for automorphic L-functions and their derivatives, Ann. of Math. 131 (1990), 53-127. MR 92e:11053

3. _ Nonvanishing theorems for L-functions of modular forms and their derivatives, Inventiones Math. 102 (1990), 543-618. MR 92a:11058

4. H. Davenport and H. Heilbronn, On the density of discriminants of cubic fields II, Proc. Roy. Soc. London Ser. A 322 (1971), 405-420. MR 58:10816

5. G. Frey, On the Selmer group of twists of elliptic curves with $\mathbb{Q}$-rational torsion points, Canad. J. Math. 40 (1988), 649-665. MR 89k:11043

6. S. Friedberg and J. Hoffstein, Nonvanishing theorems for automorphic L-functions on GL(2), Ann. of Math. 142 (2) (1995), 385-423. MR 96e:11072

7. D. Goldfeld, Conjectures on elliptic curves over quadratic fields, Number theory, Carbondale 1979 (Proc. Southern Illinois Conf., Southern Illinois Univ., Carbondale, Ill., 1979), Lecture Notes in Math, vol. 751, Springer, 1979, pp. 108-118, Berlin. MR 81i:12014

8. D. R. Heath-Brown, The size of Selmer groups for the congruent number problem I, Invent. Math. 111 (1) (1993), 171-195. MR 93j:11038

9. (2) (1994), 331-370. MR 95h:11064

10. H. Iwaniec, On the order of vanishing of modular L-series at the critical point, Sém. de Théor. Nombres, Bordeaux 2 (2) (1990), 365-376. MR 92h:11040

11. K. James, An example of an elliptic curve with a positive density of prime quadratic twists which have rank zero, Proceedings of Topics in Number Theory (G. Andrews and K. Ono, eds.), Kluwer, to appear.

12. B. Jones, The Arithmetic Theory of Quadratic Forms, Mathematical Association of America, 1950. MR 12:244a 
13. V.A. Kolyvagin, Finiteness of $E(\mathbb{Q})$ and the Tate-Shafarevich group of $E(\mathbb{Q})$ for a subclass of Weil curves, Izv. Akad. Nauk SSSR Ser. Mat 52 (1988), 522-540, 670-671. MR 89m:11056

14. J. L. Lehman, Levels of positive definite ternary quadratic forms, Math. Comp. 58 (197) (1992), 399-417, S17-S22. MR 92f: 11057

15. W. Li, Newforms and functional equations, Math. Ann. 212 (1975), 285-315. MR 51:5498

16. D. Lieman, Nonvanishing of L-series associated to cubic twists of elliptic curves, Ann. of Math. (2) 140 (1) (1994), 81-108. MR 95g:11044

17. M.R. Murty and V.K. Murty, Mean values of derivatives of modular L-series, Ann. of Math. 133 (1991), 447-475. MR 92e: 11050

18. J. Nakagawa and K. Horie, Elliptic curves with no torsion points, Proc. A.M.S. 104 (1988), 20-25. MR 89k:11113

19. K. Ono, Rank zero quadratic twists of modular elliptic curves, Compositio Math. 104 (1996), 293-304. MR 98b:11071

20. _ Twists of elliptic curves, Compositio Math. 106 (1997), 349-360. CMP 97:14

21. K. Ono and C. Skinner, Fourier coefficients of half-integral weight modular forms modulo $\ell$, Ann. of Math. (to appear).

22. _ Non-vanishing of quadratic twists of modular L-functions, Invent. Math. (to appear).

23. B. Schoeneberg, Das Verhalten von mehrfachen Thetareihen bei Modulsubstitutionen, Math. Ann. 116 (1939).

24. G. Shimura, On modular forms of half integral weight, Ann. of Math. (2) 97 (1973), 440-481. MR 48:10989

25. C. Siegel, Gesammelte Abhandlungen Bd. 3, Springer Verlag, 1966, pp. 326-405. MR 33:5441

26. J.L. Waldspurger, Sur les coefficients de Fourier des formes modulaires de poids demi-entier, J. Math. Pures. et Appl. 60 (1981), 375-484. MR 83h:10061

27. S. Wong, Rank zero twists of elliptic curves, preprint.

28. Gang Yu, Quadratic twists of a given elliptic curve over $\mathbb{Q}$, preprint.

Department of Mathematics, Pennsylvania State University, 218 McAllister Building, University Park, Pennsylvania 16802-6401

E-mail address: klj@math.psu.edu 